\title{
Electrical switching in benzidine-DDQ under pressure
}

\author{
T RAVINDRAN and S V SUBRAMANYAM \\ Department of Physics, Indian Institute of Science, Bangalore 560 012, India \\ MS received 17 November 1990; revised 16 May 1991
}

\begin{abstract}
The effect of pressure on non-ohmic conduction and electrical switching in the charge transfer complex benzidine-DDQ has been studied up to a pressure of 7.66 GPa at a temperature of $300 \mathrm{~K}$. Pulsed I-V measurements reveal heating contribution to nonohmicity and switching. At high electric fields $\left(\sim 3 \times 10^{3} \mathrm{~V} / \mathrm{cm}\right)$, the sample switches from high resistance OFF state of several kiloohms to low resistance ON state of several ohms. Temperature dependence of conductivity of $\mathrm{ON}$ state show semiconducting behaviour with very low activation energy.
\end{abstract}

Keywords. High pressure; non-ohmic conduction; electrical switching; benzidine-DDQ.

\section{Introduction}

Non-ohmic conduction and electrical switching is observed in many of the charge transfer complexes (Subramanyam and Hemamalini Naik 1985). Non-ohmic conduction observed in one-dimensional conductors TTF-TCNQ (Gunning and

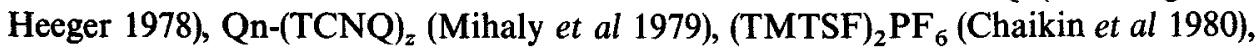
$\mathrm{NbSe}_{3}$ and $\mathrm{TaS}_{3}$ (Fleming and Grimes 1979; Miller et al 1983) and (CH) $)_{x}($ Epstein et al 1980) was explained by depinning of charge density wave (CDW) by an applied electric field. Space charge limited current (Farges et al 1972), phononassisted hopping through random barriers (Lee and Rice 1979) and tunnelling of electrons through barriers in the conducting strands (Patrick and Rowland 1979) are also used to explain non-ohmic conduction.

Electrical switching observed in quasi one-dimensional conductors is associated with the CDW conduction (Zettl and Gruner 1982; Monceau et al 1982). Other systems showing switching behaviour are amorphous chalcogenides (Csillag 1973), insulating films of mylar, anthracene (Elsharkawi and Kao 1977) and $\mathrm{Cu}-\mathrm{TCNQ}$ (Potember et al 1979).

In the present investigation, the non-ohmic conduction and electrical switching in the quasi one-dimensional charge transfer complex benzidine-2, 3-dichloro-5, 6dicyano-p-benzoquinone (benzidine-DDQ) are studied. The results can be explained by Poole-Frenkel effect, space charge limited current and charge delocalization due to heating effects. Pulsed I-V measurements are therefore employed. At high fields $\left(\sim 3 \times 10^{3} \mathrm{~V} / \mathrm{cm}\right)$ sample switches from low conducting OFF state to high conducting $\mathrm{ON}$ state with $\sigma_{\mathrm{ON}} / \sigma_{\mathrm{OFF}} \approx 10^{3}$. On withdrawing the driving current, the sample remains in the $\mathrm{ON}$ state. Temperature dependence of resistivity shows that OFF state is semiconducting whereas the ON state is weakly temperature dependent with very low activation energy.

\section{Experimental}

The charge transfer complex benzidine-DDQ was prepared by the procedure due to 
Brass and Clar (1956). The starting materials were purified by repeated crystallization. The complex in the stoichiometric ratio $1: 1$ was prepared using benzene as the solvent medium. The complex was characterized spectroscopically by the charge transfer band. The X-ray powder diffraction pattern of the sample did not give well-defined peaks. So the sample was presumed to be in the amorphous state.

Electrical measurements were carried out by two-probe technique up to a pressure of $7.66 \mathrm{GPa}$ in a tungsten carbide anvil apparatus described by Bandopadhyay et al (1980a). Electrical contacts were by pressure contact using high purity copper leads. Compacted pellets were used for electrical measurements. A Keithley 616 digital electrometer and a multilogger SC 7501 of Iwatsu company were used as measuring instruments. The dc current-voltage characteristics were determined by a circuit driven by a constant current source and voltage was measured at the same points of contact to the sample. Pulsed I-V measurements were done by keeping the OFF time of $20 \mathrm{~ms}$ constant and varying the ON time. Pulses were generated by a circuit constructed in our laboratory. Voltage across the sample and a standard resistance were measured independently by digital storage oscilloscope SS 5802 of Iwatsu company.

\section{Results and discussion}

The dependence of resistivity of benzidine-DDQ up to a pressure of $7.66 \mathrm{GPa}$ at room temperature is shown in figure 1 . Room temperature resistivity is $3.66 \times 10^{3}$ $\Omega$-cm (Matsunaga and Saito 1971). Decrease in resistance with pressure is exponential with a fall of resistance of about three orders of magnitude at $7.66 \mathrm{GPa}$ from that at ambient pressure. With increase in pressure the molecular orbitals come closer which results in fall in resistance (Bandopadhyay et al 1980b).

DC current-voltage characteristics at different pressures are shown in figure 2. As pressure increases non-ohmic conduction starts at lower fields. At two pressures of

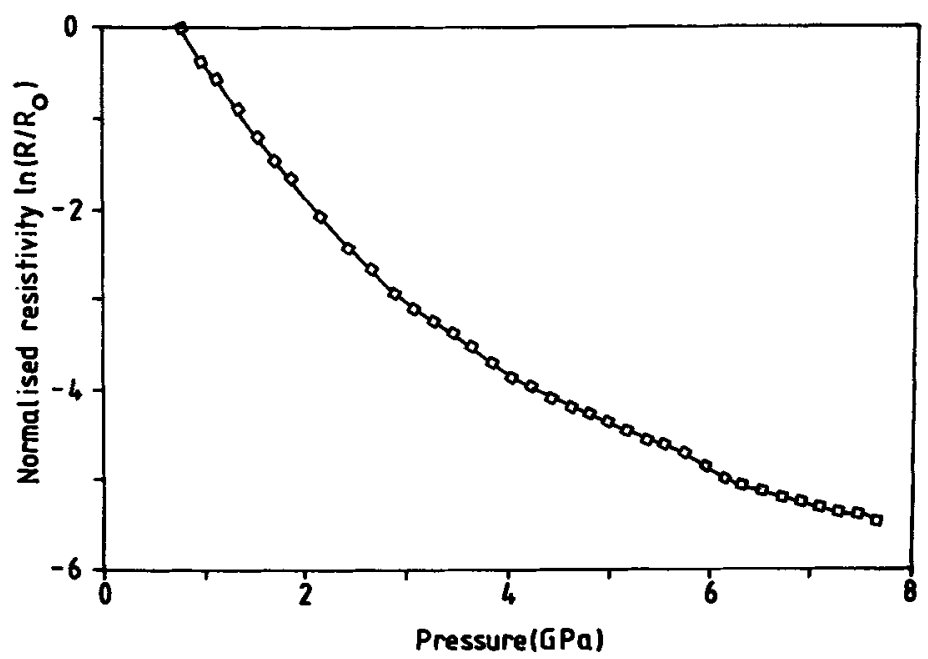

Figure 1. Pressure dependence of normalized resistivity of benzidine-DDQ, $T=300 \mathrm{~K}$. 


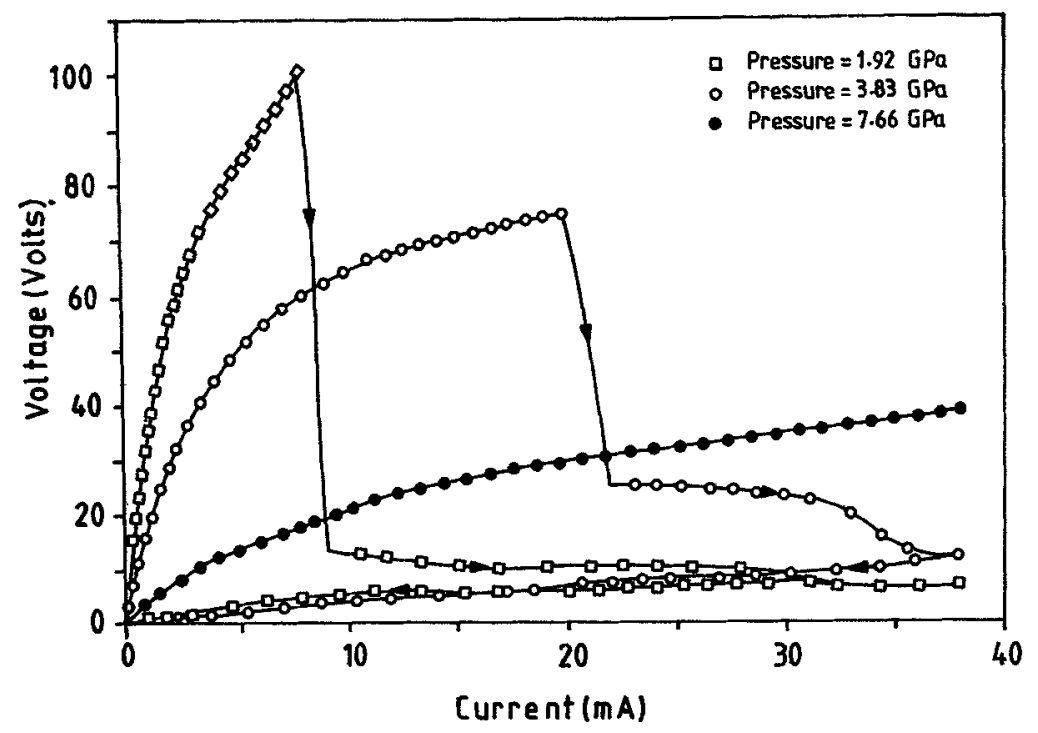

Figure 2. Current-voltage characteristics of benzidine-DDQ at different pressures, $T=300 \mathrm{~K}$.

1.92 and $3.83 \mathrm{GPa}$, the sample (typical dimensions $1 \mathrm{~mm} \times 0.25 \mathrm{~mm} \times 0.25 \mathrm{~mm}$ ) switches at a field $\sim 3 \times 10^{3} \mathrm{~V} / \mathrm{cm}$ from low conducting OFF state of several kiloohms to high conducting $\mathrm{ON}$ state of several ohms. The switching field decreases with an increase in pressure. Larger currents are needed to drive the sample to switch to $\mathrm{ON}$ state at higher pressures. The voltage difference between the threshold point for switching and the voltage at which it switches decreases with increase in pressure. Near the switching region voltage is found to be unstable. After switching, as driving current increases, differential negative resistance region with unstable voltage is observed. On reversing the current, instability in the voltage signal and differential negative resistance vanish. Even when the driving current is removed, the sample does not switch to OFF state. I-V characteristics show a hysteresis as shown in figure 3 . This reduces as the experiment is repeated. In the OFF state temperature dependence of resistivity at a clamped pressure of $3.83 \mathrm{GPa}$ (figure 4) shows semiconducting behaviour with an activation energy of $0.06 \mathrm{eV}$. Temperature dependence of resistivity of the ON state, shown in figure 4, is weakly temperature-dependent having an activation energy of $0.003 \mathrm{eV}$.

Pulsed I-V measurements (figure 5) show that keeping the OFF time $20 \mathrm{~ms}$ constant and varying the $\mathrm{ON}$ time to $100 \mu \mathrm{s}, 1 \mathrm{~ms}$ and $20 \mathrm{~ms}$ increases the nonlinearity. This reveals the heating contribution to non-ohmicity and switching field.

Benzidine-DDQ shows ohmic behaviour at low field and transition to non-ohmic region at a field $\sim 10^{3} \mathrm{~V} / \mathrm{cm}$. All current-voltage data can be normalized to a single curve (Walsh et al 1969) as shown in figure 6.

$$
\frac{I}{I_{0}(P)}=F\left[\frac{V}{V_{0}(P)}\right] \text {. }
$$

$I_{0}(P)$ and $V_{0}(P)$ are the normalizing current and the voltage respectively. 


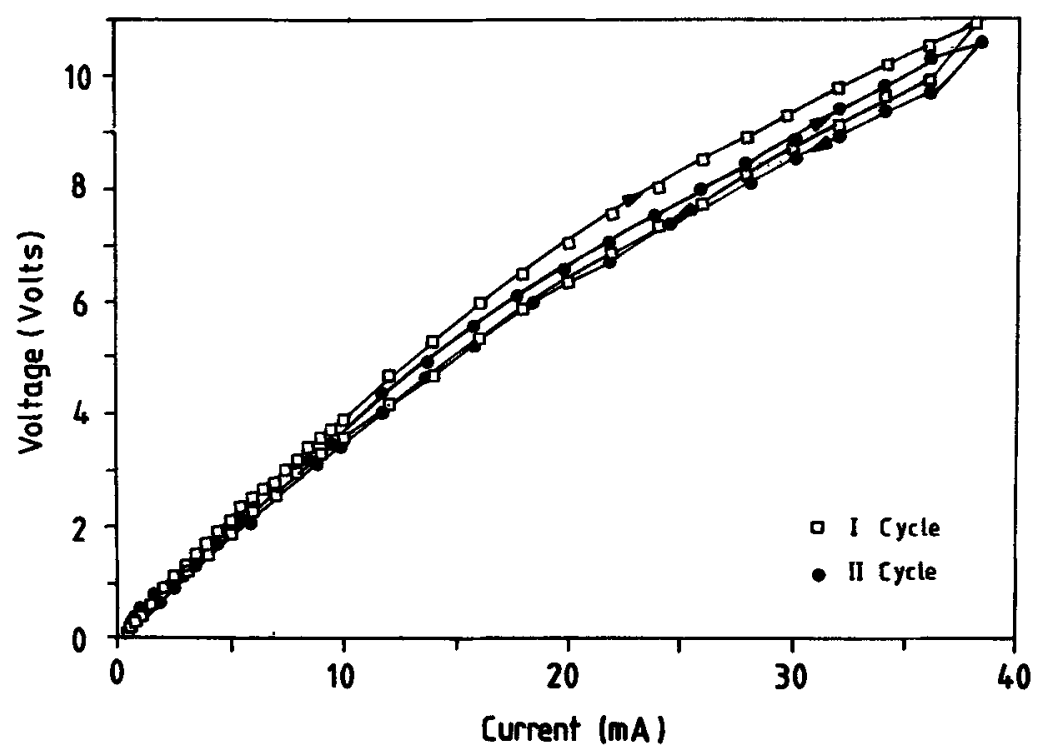

Figure 3. Current-voltage characteristics of switched sample benzidine-DDQ, $T=300 \mathrm{~K}$.

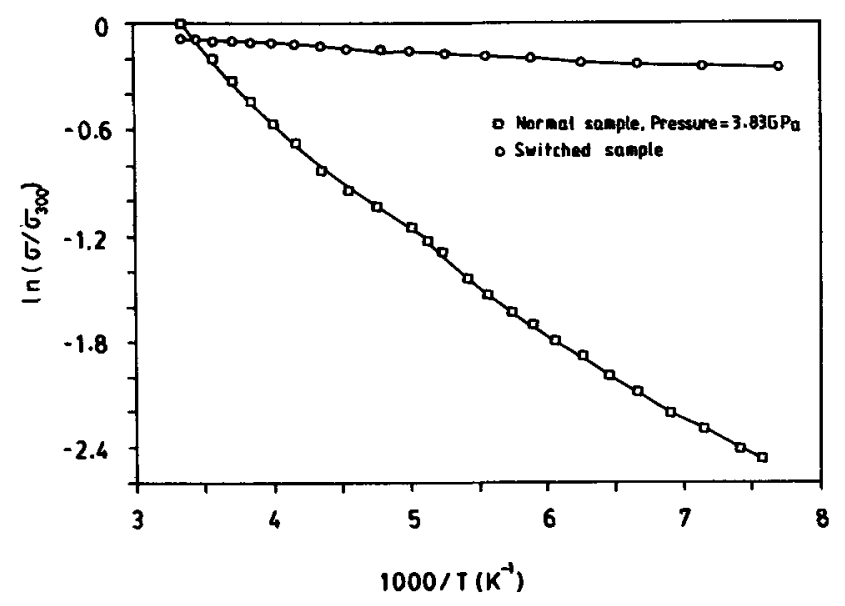

Figure 4. Temperature dependence of conductivity of normal and switched sample of benzidine-DDQ.

$\mathrm{Ln}$ - $\mathrm{Ln}$ plot of current voltage relation for the sample is shown in figure 7. At low fields the slope is found to be unity and at higher fields $\sim 10^{3} \mathrm{~V} / \mathrm{cm}$ a transition to power law region is observed. Such a power law dependence of I-V characteristics is due to crystal imperfections (Zuleeg 1963; Lampert et al 1964). Transition voltage $V_{T}$ from ohmic to non-ohmic region is a function of pressure, decreasing with an increase in pressure. For a solid free of traps, the space charge limited current density $J=x \mu V^{2} / t^{3}$ where $x$ is the dielectric constant of the medium, $\mu$ is the mobility, $t$ is the distance between the electrodes and $V$ is the applied voltage (Rose 1955; Gutman and Lyons 1967). At lower fields the current is due to, thermally- 


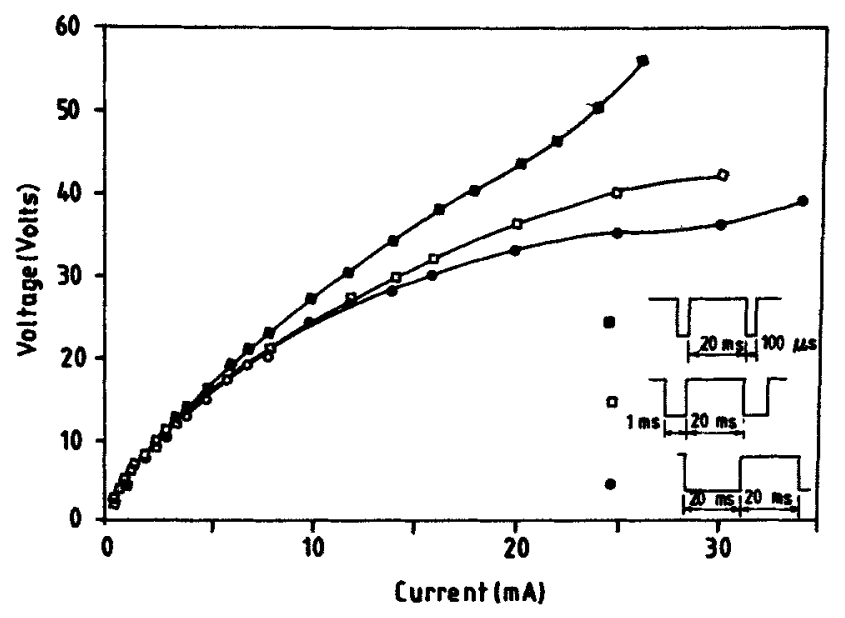

Figure 5. Pulsed current-voltage characteristics of benzidine-DDQ, Pressure $=3 \cdot 83 \mathrm{GPa}$, $T=300 \mathrm{~K}$.

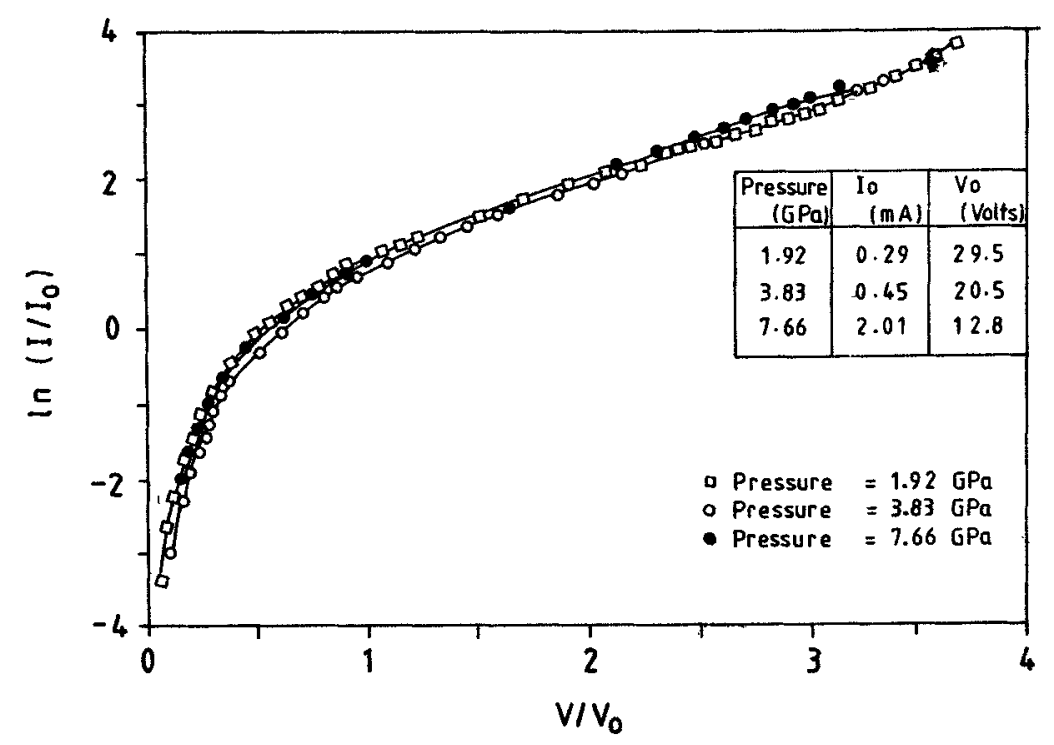

Figure 6. Normalized current-voltage characteristics of benzidine-DDQ at different pressures.

generated carriers. When the applied voltage is greater than a critical voltage, injected-free carrier concentration dominates the equilibrium thermally-generated carrier concentration. This results in space charge limited current to predominate. In the present case, the deviation from the square law behaviour is associated with the traps present in the material.

In figure 8 above a field $\sim 200 \mathrm{~V} / \mathrm{cm} \mathrm{In} I$ vs $V^{1 / 2}$ is a straight line. Non-ohmic conduction observed in this sample before switching follows the functional form of either electrode limited Schottky emission or bulk Poole-Frenkel effect. Dielectric 


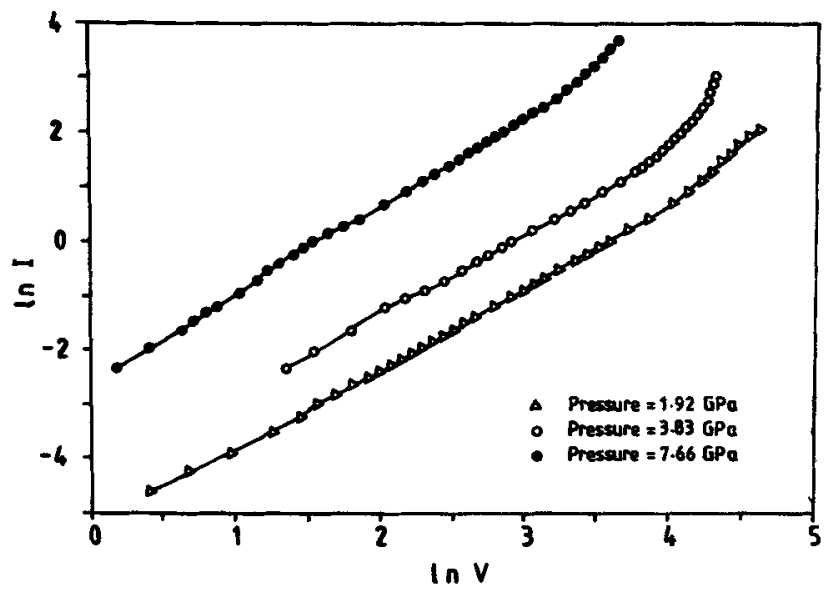

Figare 7. Plot of $\ln I$ vs $\ln V$ of benzidine-DDQ for three pressures.

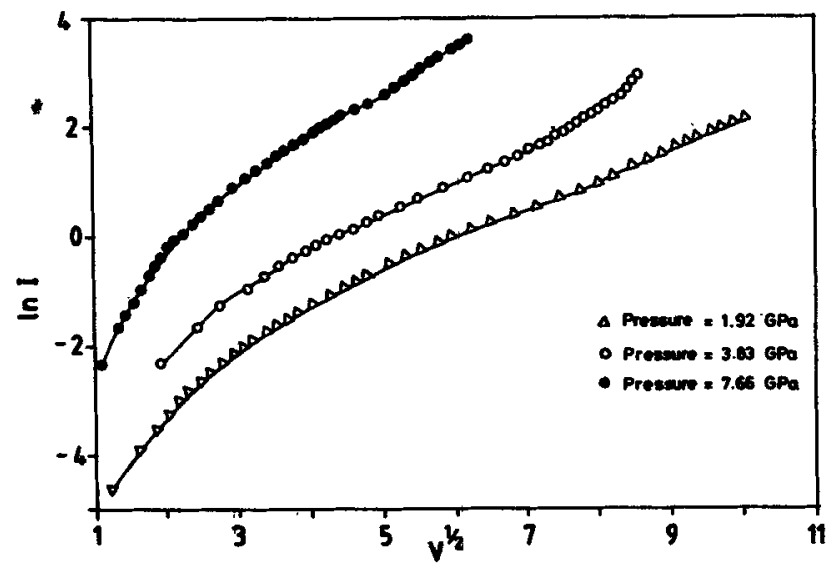

Figure 8. Plot of $\ln /$ vs $V^{1 / 2}$ of benzidine-DDQ at different pressures.

relaxation experiment has to be performed to clarify this contribution. Current $I$ due to Poole-Frenkel effect (Adkins et al 1970) is

$$
I=I_{0} \exp \frac{e\left[\beta V^{1 / 2}-V_{g}\right)}{k_{B} T} \text { with } \beta=\left(\frac{e}{\pi \varepsilon \varepsilon_{0} t}\right)^{1 / 2} \text {, }
$$

where $I_{0}$ is a constant, $V_{g}$ the energy difference between the trap level and the bottom of the conduction band, $\varepsilon_{0}$ the permittivity of free space, $\varepsilon$ the relative dielectric permittivity, $T$ the absolute temperature, $k_{B}$ the Boltzmann constant and $e$ the charge of the electron. Poole-Frenkel effect is the field-assisted thermal ionization of electrons from the discrete trap level into the conduction band due to the lowering of the Coulombic potential barrier by an applied electric field. A positively charged trap, i.e. a trap that is positively charged when empty and uncharged when it is filled, gives rise to Coulombic potential barrier. A donor or acceptor centre can also manifest in Poole-Frenkel effect. 
Non-ohmicity observed in the pulsed experiment can arise from thermal ionization from impurities and transition of electrons from valence band to conduction band thereby enhancing the current. In brief, we have observed fieldinduced phase transition similar to that of $o$-tolidine-iodine (Hemamalini Naik and Subramanyam 1986).

\section{Conclusion}

At high electric fields $\left(\sim 3 \times 10^{3} \mathrm{~V} / \mathrm{cm}\right)$, switching is observed in the charge transfer complex benzidine-DDQ under pressure with $\sigma_{\mathrm{ON}} / \sigma_{\mathrm{OFF}} \approx 10^{3}$ at a temperature of $300 \mathrm{~K}$. Non-ohmic conduction can arise from either space charge limited current or Poole-Frenkel effect. Pulsed I-V measurements show heating contribution to nonohmic conduction and switching. The $O N$ state is stable even after removing the driving field and it is semiconducting with very low activation energy.

\section{Acknowledgement}

Authors are thankful to the Department of Science and Technology for financial support.

\section{References}

Adkins C J, Freake S M and Hamilton E M 1970 Philos. Mag. 22183

Bandopadhyay A K, Chatterjee S, Subramanyam S V and Bulka B R 1980a J. Phys. C13 L803

Bandopadhyay A K, Nalini A V, Gopal E S R and Subramanyam S V 1980b Rev. Sci. Instrum. 51136 Brass K and Clar E 1956 Bull. Chem. Soc. Jpn. 29213

Chaikin P M, Gruner G, Engber E M and Greene R L 1980 Phys. Rev. Lett. 451874

Csillag A 1973 in Electronic and structural properties of amorphous semiconductors (eds) P G Le Camber and J Mort (Orlando: Academic Press) p. 319

Elsharkawi A R and Kao K C 1977 J. Phys. Chem. Solids 3895

Epstein A J, Gibson H W, Chaikin P M, Clark W G and Gruner G 1980 Phys. Rev. Lett. 451730

Farges J P, Brass A and Gutman F 1972 J. Phys. Chem. Solids 331723

Fleming R M and Grimes C C 1979 Phys. Rev. Lett. 421423

Gunning. W J and Heeger A J 1978 Solid State Commun. 27843

Gutman F and Lyons L E 1967 Organic semiconductors (New York: Wiley) ch. 10

Hemamalini Naik and Subramanyam S V 1986 Pramana-J. Phys. 2661

Lampert M A, Many A and Mark E 1964 Phys. Rev. 135 A1444

Lee P A and Rice T M 1979 Phys. Rev. B19 3970

Matsunaga Y and Saito G 1971 Bull. Chem. Soc. Jpn 44958

Mihaly G, Janossy A, Kurti A, Ferro J and Gruner G 1979 Phys. Status Solidi B94 284

Miller J H Jr, Richard J, Tucker J R and Bardeen J 1983 Phys. Rev. Lett. 511592

Monceau P, Richard J and Renard M 1982 Phys. Rev. B25 931

Patrik M L and Rowland T J 1979 Phys. Rev. Lett. 43879

Potember R S, Poehler T O and Cowan D O 1979 Appl. Phys. Lett. 34405

Rose A 1955 Phys. Rev. 971538

Subramanyam S V and Hemamalini Naik 1985 in The metallic and non-metallic states of matter: An important facet of chemistry and physics of the condensed state (eds) P P Edwards and C N R Rao (London and Philadelphia: Taylor and Francis)

Walsh P J, Vogel R and Evans E J 1969 Phys. Rev. 1781274

Zettl A and Gruner G 1982 Phys. Rev. B26 2298

Zuleeg R 1963 Solid State Electron. 6645 